\title{
Helicobacter pylori-negative intestinal-type gastric adenoma successfully treated by endoscopic submucosal dissection: a case report
}

Authors

Institutions
Yoshiya Kobayashi ${ }^{1}$, Yoshinori Komazawa ${ }^{1}$, Makoto Nagaoka' ${ }^{1}$, Yoshiko Takahashi ${ }^{1}$, Mika Yuki $^{1}$, Toshihiro Shizuki ${ }^{1}$, Toru Nabika

${ }^{1}$ Izumo City General Medical Center - Internal Medicine, Izumo, Shimane, Japan

${ }^{2}$ Shimane University School of Medicine - Functional Pathology, Izumo, Shimane, Japan submitted 31. January 2016 accepted after revision 5. July 2016

\section{Bibliography}

Dol http://dx.doi.org/

10.1055/s-0042-112583

Published online: 8.8.2016

Endoscopy International Open 2016; 04: E986-E989

(c) Georg Thieme Verlag KG

Stuttgart · New York

E-ISSN 2196-9736

\section{Corresponding author}

Yoshiya Kobayashi

Izumo City General Medical

Center

Internal Medicine

613 Nadabun-cho

Izumo-Shi Shimane 691-0003

Japan

Phone: +81-853-63-5111

Fax: +81-853-63-4228

yoshiya_kobayashi@hotmail. com
Background and study aims: A 49-year-old woman underwent an esophagogastroduodenoscopy as part of a health check at our hospital. Endoscopic observation revealed a flat elevated lesion $6 \mathrm{~mm}$ in diameter in the gastric antrum (Paris Classification type Ila). Magnifying endoscopy using narrow-band imaging showed a slightly irregular micro-surface pattern with round and oval pits, as well as a regular micro-vascular pattern without a demarcation line. Atrophy and intestinal metaplasia were not recognized in the background gastric mucosa. Furthermore, Helicobacter pylori infection was not detected by histologic, serologic, and urea breath test results.

\section{Introduction}

$\nabla$

Gastric adenoma is a neoplastic lesion characterized by localized and polypoid proliferation of dysplastic epithelium. In affected patients, in addition to a higher probability of an adenocarcinoma found elsewhere in the stomach, the adenoma itself has potential to progress to an adenocarcinoma [1,2]. Gastric adenomas are histologically classified as intestinal-type, gastric-type, and indeterminate type, of which intestinal-type is the most prevalent. An intestinal-type adenoma is usually found with a background of intestinal metaplasia commonly caused by infection with Helicobacter pylori (H. pylori), widely known as a carcinogenic bacterium, thus patients with a diagnosis of $H$. pylori-associated gastritis should be followed with endoscopic surveillance. On the other hand, $H$. pylori-negative patients are considered to have a lower risk of gastric neoplasms. Herein, we report a rare case of gastric adenoma in a patient without evidence of intestinal metaplasia or H. pylori infection.
Endoscopic resection was performed for histologic evaluation, and a pathologic diagnosis of intestinal-type gastric adenoma occurring in pyloric mucosa without atrophy or metaplasia was established.

Immunohistochemistry findings of the lesion showed the intestinal epithelium phenotype with positive staining for MUC2, CD10, and CDX2. Furthermore, irregular distribution with a higher positive proportion of Ki-67 was found in the lesion, indicating its malignant potential. We report here a rare case of gastric adenoma without surrounding intestinal metaplasia occurring in a Helicobacter pylori-negative patient.

\section{Case report}

A 49-year-old woman underwent an esophagogastroduodenoscopy (EGD) examination as part of a health check at our hospital, which revealed a flat elevated lesion $6 \mathrm{~mm}$ in diameter in the gastric antrum (Paris Classification IIa) ( $\bullet$ Fig. 1a). Chromoendoscopy with $0.1 \%$ indigo carmine also clearly showed a reddened area ( $\bullet$ Fig. $\mathbf{1 b}$ ), while magnifying endoscopy using narrow-band imaging (ME-NBI) revealed a slightly irregular micro-surface pattern with round and oval pits (- Fig.2). According to the vascular surface classification system reported by Yao et al. [3], the ME-NBI findings were categorized as a regular microvascular pattern and slightly irregular micro-surface pattern without a demarcation line. Hence, it was diagnosed as a non-cancerous lesion and biopsy specimens indicated intestinaltype gastric adenoma. Atrophy, intestinal metaplasia, spotty erythema, and edema were not recognized in the background gastric mucosa, while a regular arrangement of collecting venules (RAC), a well-known characteristic endoscopic finding in $H$. pylori-negative patients [4], was clearly found in the gastric corpus. These endoscopic findings strongly indicated $H$. pylori-nega- 

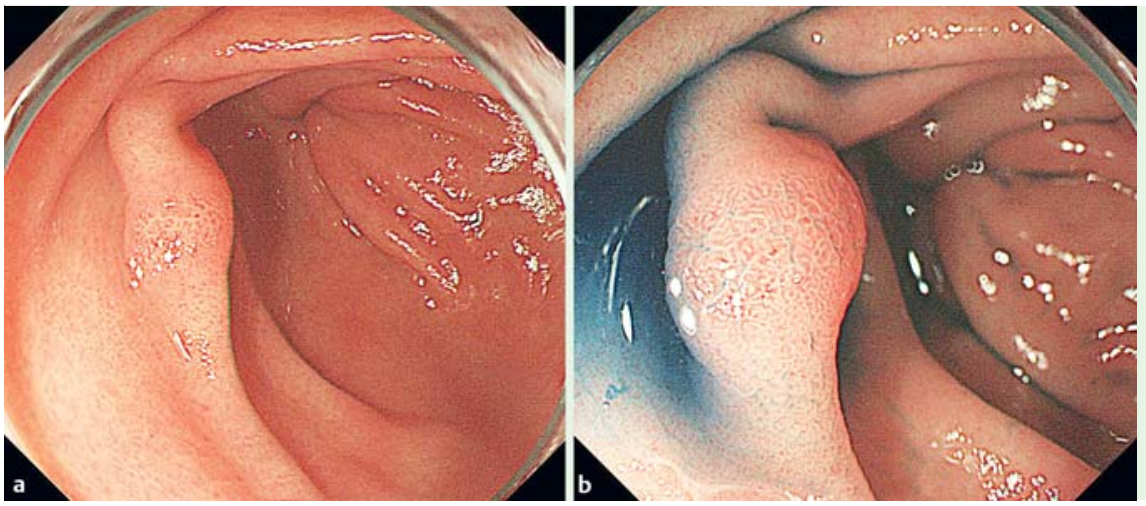

Fig. 1 Endoscopic images of the lesion. a Conventional white-light imaging showed a reddish colored slightly elevated lesion $6 \mathrm{~mm}$ in diameter located in the gastric antrum. $\mathbf{b}$ Chromoendoscopy using $0.1 \%$ indigo carmine dye showed a clearly defined flat elevated lesion with a slightly irregular surface pattern.

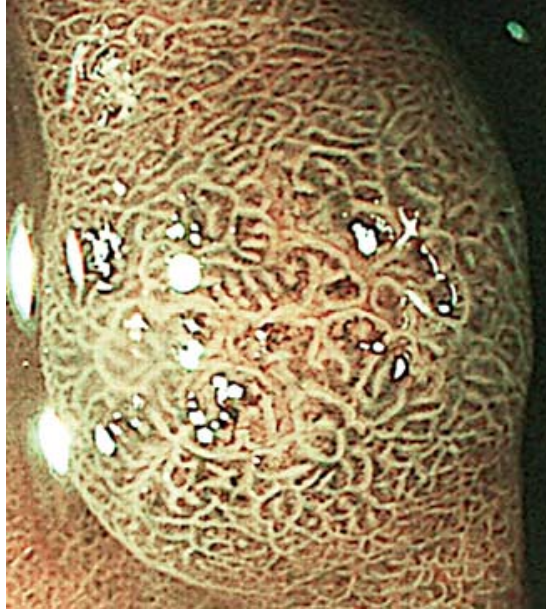

Fig. 2 Magnifying endoscopy with narrow-band imaging. We observed a slightly irregular micro-surface pattern and regular micro-vessel pattern with an unclear demarcation line.

tive status. Furthermore, serological examinations revealed antiH. pylori IgG antibodies at $4.3 \mathrm{U} / \mathrm{mL}(<10 \mathrm{U} / \mathrm{mL})$, pepsinogen (PG) I at $66.3 \mathrm{ng} / \mathrm{dL}$ (>70 ng/dL), and a PGI/II ratio of $5.5(\mathrm{PGI} / \mathrm{II}>3.0)$ in serum, while a urea breath test was $0.3 \%$ 。 $(<2.5 \%$ o).

An endoscopic resection was performed for a detailed histologic evaluation and the lesion was removed en bloc without complications. We generally perform endoscopic submucosal resection (ESD), as this technique has a higher rate of successful en bloc resection (5) and can provide a reliable pathological diagnosis.
Although no intestinal metaplasia or $H$. pylori bacteria were noted, and normal foveolar epithelium was found in the background mucosa, a histologic diagnosis of intestinal-type highgrade gastric adenoma was established based on the WHO criteria ( $\bullet$ Fig.3a, $\square$ Fig.3b, $\bullet$ Fig.3c). Together, endoscopic, serologic, and histologic results led us to conclude that the lesion occurred in true $H$. pylori-negative gastric mucosa. In addition, immunohistochemistry findings demonstrated an intestinal cell phenotype with positive staining for MUC2, CD10, and CDX2, whereas MUC5AC and MUC6 were negative ( $\bullet$ Fig.4a, $\bullet$ Fig.4b $\checkmark$ Fig.4c, $\odot$ Fig.4d, $\odot$ Fig.4e). Furthermore, the lesion showed an irregular distribution, though with a higher positive proportion of Ki-67, indicating a relatively high malignant potential (৫ Fig. 4f), while p53 immunohistochemistry findings revealed scattered-type staining, which did not indicate an abnormality

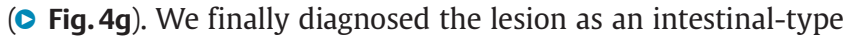
gastric adenoma occurring in normal pyloric mucosa without intestinal metaplasia in an $\mathrm{H}$. pylori-negative patient, a rare case.

\section{Discussion}

Gastric adenomas (low-grade intraepithelial neoplasia, lowgrade dysplasia), a type of benign noninvasive intraepithelial neoplasia, are considered to be premalignant lesions $[1,2]$. Most occur in the background of atrophic gastritis or intestinal meta-
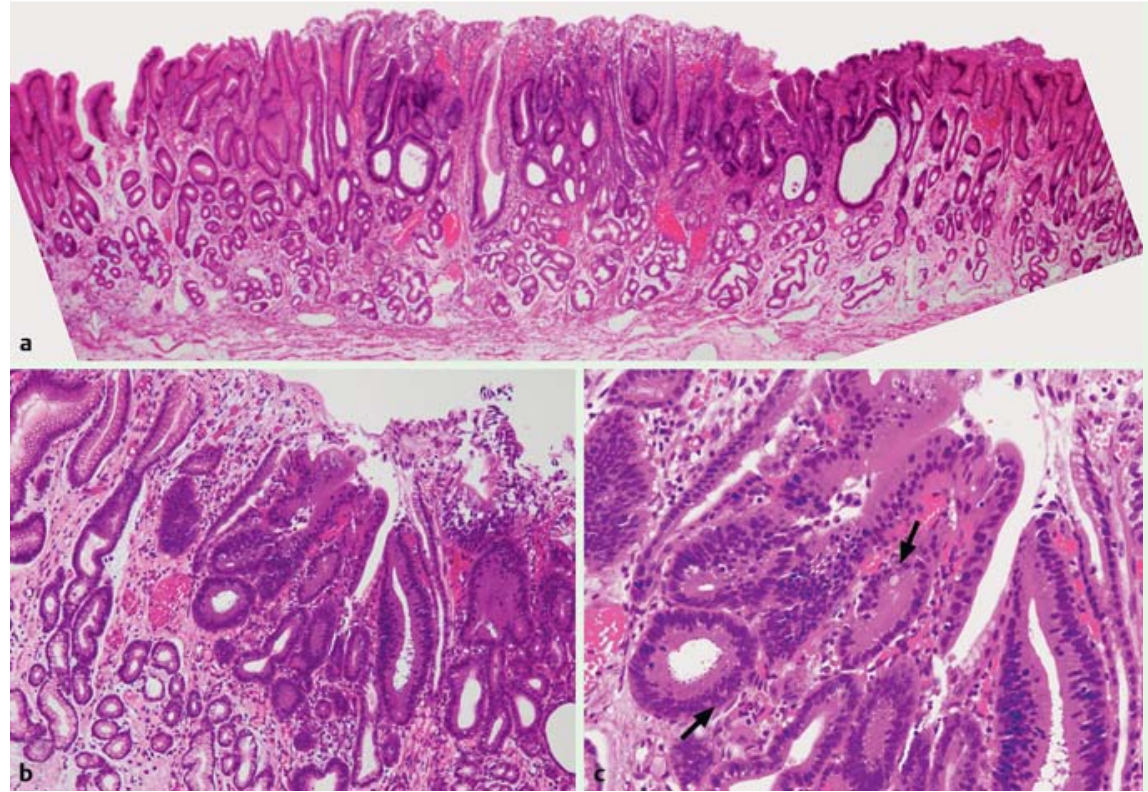

Fig. 3 Histologic appearance of lesion (HE staining). a On low-power view $(\times 20)$, the lesion was slightly elevated and showed a tubular structure with slight structural atypia, suggesting an intestinal-type gastric adenoma. No metaplasia was seen in the background mucosa. b, $\mathbf{c}$ On high-power views $(\mathbf{b} \times 100, \mathbf{c} \times 200)$, the lesion had histologic characteristics of intestinal epithelium with goblet cells (arrows) and round hyperchromatic nuclei. H. pylori organisms were not seen in the Giemsastained specimen (not shown). 


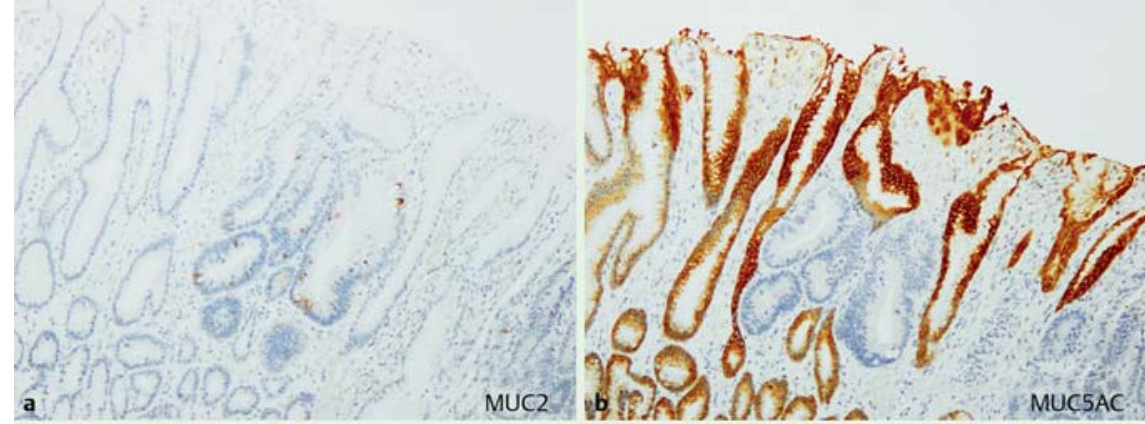

Fig.4 Immunohistochemical examination for gastric or intestinal phenotype, and expression of Ki-67 and p53. The lesion was positive for (a) MUC2, (d) CD10, and (e) CDX2, and negative for (b) MUC5AC and (c) MUC6, indicating that the neoplastic epithelium was the intestinal phenotype. $\mathrm{f} \mathrm{Ki-67}$ was highly positive, while $(\mathbf{g})$ p53 showed scatteredtype staining, which indicated no abnormality in the neoplastic lesion.

\section{.}
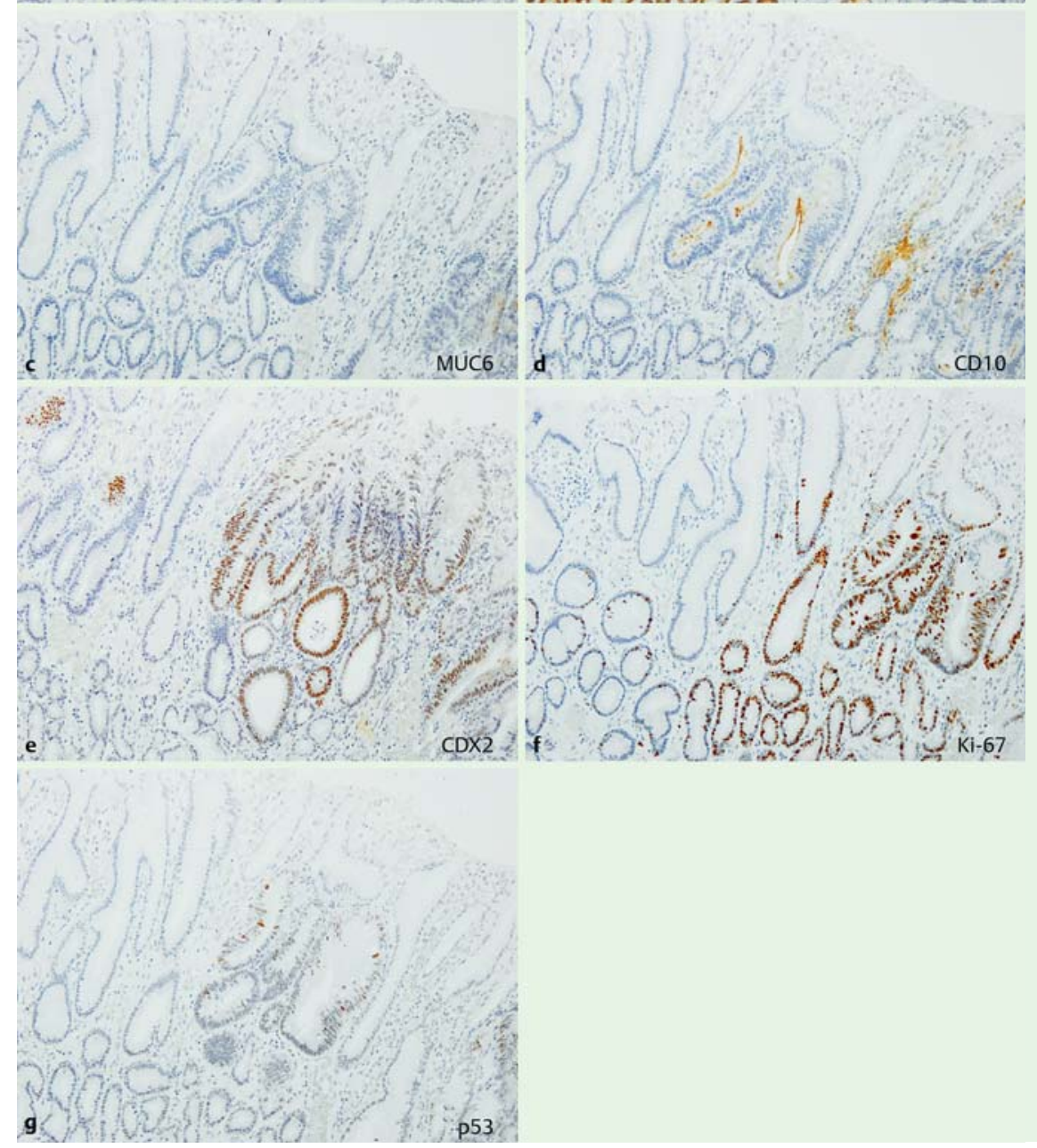

plasia, and are commonly found in patients with $H$. pylori infection. Because of their involvement in the pathological process of adenocarcinoma development [1,2], endoscopic resection is usually chosen for treatment [6].

Recently, ME-NBI was introduced as a useful tool for management of gastric adenomas [7]. In addition, histologic examination of expressed mucin in such lesions is becoming popular as a new diagnostic method to evaluate their biological behavior. Gastric neoplasms are classified into gastric-phenotype, intestinal-phenotype, and indeterminate, based on expression of the human gastric mucin markers MUC2, MUC5AC, MUC6, and CD10 [8]. At our institution, we use MUC5AC as an immunohistochemical marker for foveolar cells, MUC6 for mucous neck or pyloric gland cells, MUC2 for goblet cells, CD10 for the intestinal brush border, and CDX2 for intestinal differentiation. In general, the gastricphenotype is classified based on expression of MUC5AC and MUC6, while the intestinal-phenotype is defined by positive ex- pression of MUC2, CD10, and CDX2. On the other hand, proliferative activity and malignant potential are assessed by expression of Ki-67 and p53.

The current lesion was positive for MUC2, CD10, and CDX2, whereas it was negative for MUC5AC and MUC6, indicating intestinal-type. In addition, its malignant potential was considered to be relatively high because of the irregular distribution and higher positive proportion of Ki-67. There was no evidence of $H$. pylori infection in the background mucosa shown in histological and serological examination findings, and endoscopy revealed no atrophic changes. As described above, this case met all criteria proposed by Matsuo T, et al. for $H$. pylori-negative gastric cancer [9]. Finally, we diagnosed the current gastric neoplasm as occurring in true $H$. pylori-negative gastric mucosa.

Previous investigators have reported gastric-type adenomas and gastric adenocarcinomas with chief-cell differentiation in $H$. pylori-negative patients. To the best of our knowledge, this is the 
first report of an intestinal-type gastric adenoma occurring in an $H$. pylori-negative patient. Some reports of $H$. pylori-negative gastric cancer (HpNGC) have been presented, although the prevalence differs, because diagnostic criteria for HpNGC have yet to be definitively established. According to a report that used the strict criteria of Matsuo et al. [9], the prevalence of HpNGC was $0.66 \%$ of all gastric cancer patients, while $66.7 \%$ of the HpNGC cases were undifferentiated type in histological findings. Therefore, the current case of $H$. pylori-negative intestinal-type differentiated adenocarcinoma is rare, though Ozaki. et al. [10] recently reported an affected patient.

In the current case, our histologic examination revealed neither $H$. pylori infection nor intestinal metaplasia in the background gastric mucosa, thus we suspected that the lesion underwent de novo progression instead of the common pathway associated with $H$. pylori infection. Its high malignant potential, as indicated by Ki-67 staining, in spite of lower histologic atypism may have been due to this uncommon pathogenesis. Although we diagnosed the lesion as high-grade dysplasia/adenoma based on the WHO criteria, other pathologists in Asian countries may make a diagnosis of well-differentiated adenocarcinoma. Diagnostic discrepancies between Asian and Western pathologists for gastric intraepithelial neoplasia are considered to be problematic, because of different terminology, diagnostic criteria, and grading systems. The Vienna classification was developed for use as common terminology throughout the world, although it has yet to befully implemented.

\section{Conclusions}

\section{$\nabla$}

In summary, we report a rare case of intestinal-type gastric adenoma in an $H$. pylori-negative patient without intestinal metaplasia. It is important for gastroenterologists to keep in mind that intestinal-type gastric adenomas can be found in patients negative for $H$. pylori infection. Further investigations of biological differences between adenomas in mucosa with and without intestinal metaplasia, as well as $H$. pylori infection are warranted.
Competing interests: None

\section{Acknowledgements \\ $\nabla$}

The authors appreciate the helpful advice about this case report that Dr. Kinoshita, Professor in the Department of Gastroenterology and Hepatology at Shimane University, provided.

\section{References}

1 De Vries AC, van Grieken NC, Looman CW et al. Gastric cancer risk in patients with premalignant gastric lesions: a nationalwide cohort study in the Netherland. Gastroenterology 2008; 134: 945

2 Lansdown M, Quirke P, Dixon MF et al. High grade dysplasia of the gastric mucosa: a marker for gastric carcinoma. Gut 1990; 31: 977

3 Yao $K$. The endoscopic diagnosis of early gastric cancer. Ann Gastroenterol 2013; 26: 11-22

4 Yagi K, Nakamura A, Sekine A. Characteristic endoscopic and magnified endoscopic findings in the normal stomach without Helicobacter pylori infection. J Gastroenterol Hepatol 2002; 17: 39-45

5 Park YM, Cho E, Kang HY et al. The effectiveness and safety of endoscopic submucosal dissection compared with endoscopic mucosal resection for early gastric cancer: a systematic review and metaanalysis. Surg Endosc 2011; 25: 2666-2677

6 Kato M, Nishida T, Tsutsui S et al. Endoscopic submucosal dissection as a treatment for gastric noninvasive neoplasia: a multicenter study by Osaka University ESD Study Group. J Gastroenterol 2011; 46: 325 - 333

7 Tsuji Y, Ohata K, Sekiguchi M et al. Magnifying endoscopy with narrowband imaging helps determine the management of gastric adenoma. Gastric Cancer 2012; 15: 414-418

8 Tajima Y, Shimoda T, Nakanishi Y et al. Gastric and intestinal phenotypic marker expression in gastric carcinomas and its prognostic significance: immunohistochemical analysis of 136 lesions. Oncology 2001; 61: $212-220$

9 Matsuo T, Ito M, Takata S et al. Low prevalence of Helicobacter pylorinegative gastric cancer among Japanese. Helicobacter16: 2011415419

10 Ozaki Y, Suto H, Nosaka T et al. A case of Helicobacter pylori-negative intramucosal well-differentiated gastric adenocarcinoma with intestinal phenotype. Clin J Gastroenterol 2015; 8: 18-21 